Mappemonde

Revue trimestrielle sur l'image géographique et les formes du territoire

$127 \mid 2019$

Varia

\title{
M. H. Edney, Cartography: The Ideal and Its History
}

The University of Chicago Press, Londres, 324 p., 40 pages de

bibliographie (!), 17 pages d'index

\section{Laurent Jégou}

\section{OpenEdition}

\section{Journals}

Édition électronique

URL : http://journals.openedition.org/mappemonde/1711

DOI : 10.4000/mappemonde.1711

ISSN : 1769-7298

Éditeur

UMR ESPACE

Référence électronique

Laurent Jégou, « M. H. Edney, Cartography: The Ideal and Its History», Mappemonde [En ligne], 127| 2019, mis en ligne le 01 juillet 2019, consulté le 25 septembre 2020. URL : http://

journals.openedition.org/mappemonde/1711; DOI : https://doi.org/10.4000/mappemonde.1711

Ce document a été généré automatiquement le 25 septembre 2020

\section{(c) (i) (3)(2)}

La revue Mappemonde est mise à disposition selon les termes de la Licence Creative Commons Attribution - Pas d'Utilisation Commerciale - Partage dans les Mêmes Conditions 4.0 International. 


\section{H. Edney, Cartography: The Ideal and Its History}

The University of Chicago Press, Londres, 324 p., 40 pages de bibliographie (!), 17 pages d'index

Laurent Jégou

\section{RÉFÉRENCE}

Edney M. H. (2019). Cartography: The Ideal and Its History. The University of Chicago Press, Londres, $324 \mathrm{p}$. 
1 L'ouvrage, paru en avril dernier, ne manquera pas d'avoir un impact sur le domaine de la recherche en cartographie, comme semblent l'indiquer les comptesrendus qui apparaissent, pour plusieurs raisons que nous allons essayer de présenter. Tout d'abord, on peut considérer ce livre comme un aboutissement des travaux d'un grand spécialiste de l'histoire de la cartographie et de son épistémologie, Matthew $\mathrm{H}$. Edney. Les idées présentées ici ont été en partie publiées précédemment, mais dans divers articles scientifiques, sur plusieurs années. Ce géographe anglo-américain a surtout travaillé en histoire de la cartographie, il est professeur à l'université du Maine du Sud (à Portland, USA), à la bibliothèque Osher de

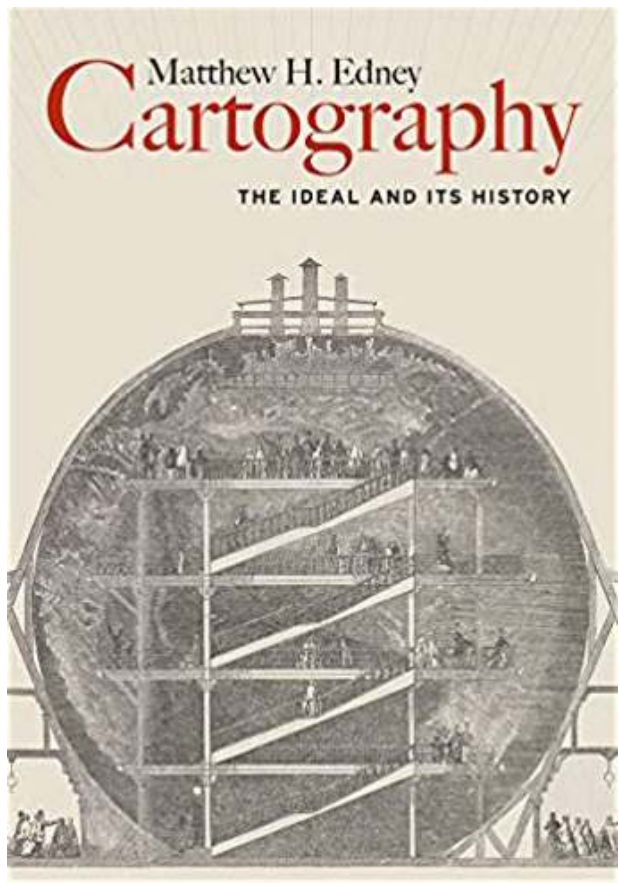
cartographie (https://oshermaps.org/).

Après avoir co-dirigé le volume 4 de l'immense projet d'Histoire de la Cartographie avec Mary Pedley (https://geography.wisc.edu/histcart/), il en devient le directeur en 2005. C'est un chercheur actif dans la communauté anglophone et internationale, on peut retrouver ses productions et ses activités sur son blog (https:// www.mappingasprocess.net/). Ensuite, et surtout, ce livre devrait marquer la réflexion sur la cartographie de par son côté provocateur, la remise en cause de nombre de préconceptions qu'il expose en détail et avec force arguments : c'est une puissante invitation à la réflexion sur nos pratiques et à poursuivre le travail engagé. Enfin, c'est un ouvrage d'une grande qualité d'écriture, clairement et logiquement structuré, qui nous guide progressivement dans l'argumentation après nous avoir exposé, dans les premiers chapitres, la motivation et le cœur du raisonnement. Ne serait-ce que par son extensive bibliographie et la large revue de littérature qu'il présente, de plus très récente, l'ouvrage est à conseiller à tous les chercheurs.

2 L'argument principal de l'ouvrage tient en une critique de la recherche en cartographie, il est exposé dès la première page sous une forme concise :

"The study of maps and mapping is bedeviled by a profound act of cultural misdirection. Modern culture deploys an idealized conception of mapping that obscures the myriad ways in which people actually go about producing, circulating, and consuming maps, whether in the past or the present. The actual behavior, what people do, is mapping. The idealized behavior, what people think they do, is cartography. »

3 Le lecteur ou la lectrice francophone ne manqueront pas de supposer que cette réflexion est facilitée en anglais, où deux noms existent pour évoquer la production de cartes : mapping et cartography; c'est justement sur la différence entre ces deux sens que se trouve l'enjeu. Conséquemment, on pourra en déduire que «l'idéal cartographique » est d'autant plus masqué dans les langues, comme le français, qui ne possèdent qu'un seul terme. 
4 L'argument de M.H. Edney tient dans l'idée que la recherche sur la cartographie est subtilement dévoyée par un ensemble de préconceptions qui forment une sorte d'idéal, qui s'est développé progressivement et qui engendre toute une série de conséquences néfastes, dont l'une des principales est un éloignement par rapport à la réalité concrète de l'utilisation, la diffusion et la consommation des cartes, dans le passé comme actuellement. L'idéal cartographique est un système culturel de croyances envahissant ("pervasive ») et méconnu (p. 4) qui tourne autour de l'idée qu'il existe une catégorie sans équivoque d'objets appelés " cartes ", tous du même type, qui, quelles que soient leurs formes et leurs fonctions, peuvent être décrits avec le même vocabulaire et évalués selon les mêmes standards. La diversité des cartes est ramenée à une fonction unique de représentation de réalités spatiales, tout ce qui s'en éloigne étant écarté de l'appellation officielle de "carte». L'idéal est formé par "un réseau compact et résilient de préconceptions qui se renforcent mutuellement, promouvant l'idée que la cartographie est une activité universelle, consistant à retranscrire le monde dans l'objectif partagé d'améliorer la civilisation par le perfectionnement d'une archive des connaissances spatiales, par l'utilisation des techniques universelles d'observation et de communication » (p. 5).

5 Le premier chapitre expose cette idée de départ et forme une introduction à l'ouvrage, il déstabilise assez pour intriguer et offre une première série de pistes de réflexions qui seront approfondies dans les chapitres suivants.

6 D'un point de vue épistémologique, l'auteur souhaite mettre en place une vision réflexive par rapport à la critique en cartographie, qu'il décompose en deux approches depuis les premiers développements scientifiques des années 1960 sur la nature des cartes (A. H. Robinson, J. Bertin). Tout d'abord, ce qu'il nomme la critique normative, qui vise à renforcer l'idée d'une norme cartographique, en critiquant ce qui s'en éloigne (et on sait les opportunités que la complexité et l'évolution des moyens de la production des cartes ont offert pour l'apparition de travaux critiquables...). Ensuite, la critique "socio-culturelle ", expression à laquelle il arrive pour ne pas tomber dans des biais interprétatifs (ou des appellations trop connotées comme le post-modernisme), qui regroupe les réflexions sur le rôle, le sens social et culturel des cartes, notamment de support des pouvoirs de contrôle des territoires, mais aussi sur ce que l'idéal a dénigré comme hétérodoxe ou anormal, à la suite d'auteurs comme J.-B. Harley, D. Woodward ou D. Wood. M. H. Edney souhaite démontrer que même la critique socioculturelle, si elle va dans le bon sens, reste victime de certaines des préconceptions liées à l'idéal cartographique et ne va pas assez loin. Dès le premier chapitre, on est donc déstabilisé et encouragé à poursuivre cette lecture stimulante.

7 Le deuxième chapitre expose les deux idées principales, les deux outils conceptuels, qui permettent de remettre en cause la notion centrale de l'idéal, l'universalité cartographique : les modes cartographiques et le renouveau de la réflexion sémiotique sur la carte comme un "document ouvert", au sens linguistique. Ces deux idées expliquent l'approche développée par l'auteur d'une cartographie vue d'abord comme une démarche, un processus (plutôt que la simple production d'objets matériels). Premièrement, l'auteur rappelle qu'il n'y a jamais eu une seule façon de concevoir les connaissances spatiales, d'utiliser les outils qui permettent de les manipuler, de les diffuser, ainsi que de les préserver. Que ce soit dans l'histoire ou dans les pratiques actuelles, il a toujours existé une diversité de formes de pratiques liées aux cartes (de mapping), qui sont minimisées par les proclamations d'universalité de l'idéal 
cartographique (centrée sur une vision culturelle occidentale et positiviste). L'auteur propose de réfléchir alors en termes de modes ou modalités cartographiques, de façons de faire, d'utiliser, et de diffuser des cartes. On comprend ici que l'auteur promeut une vision dynamique et complexe des cartes : il s'agit de processus, de démarches, multiples, pas seulement des documents finaux. Cette approche permet alors de distinguer des " arrangements", des configurations de processus: des modes. Le besoin d'une plus grande prise en compte de l'utilisation des cartes, de leur consommation, résonne avec les propositions récentes de la cartographie critique et de la sémiotique visuelle (à la suite de l'histoire de l'art et de l'esthétique qui s'intéressent à la réception depuis longtemps, pourrait-on remarquer).

Ici l'auteur propose un exemple éclairant : ce n'est pas parce que les moyens techniques de gestion et de représentation des données spatiales se sont progressivement concentrés autour des Systèmes d'Information Géographiques que tous les utilisateurs produisent les mêmes cartes, de la même manière, pour les mêmes objectifs et utilisateurs. Si le degré d'unité technique s'est accru, il n'a pas transformé les conditions sociales qui induisent des modes cartographiques variés de circulation et de consommation des cartes.

Un tableau, page 33, décrit les 14 modes repérés par l'auteur au fil de ses travaux en histoire de la cartographie, regroupés en trois familles :

- Représentation fine de portions discrètes du monde, potentiellement observables par un individu (paysages, villes, propriétés, planification d'infrastructures...)

- Représentations moins fines d'espaces plus grands, dont la taille les rend inaccessibles à l'observation par un seul individu (cosmogonies, globe, cartes marines ou célestes)

- Les représentations modernes produites par les États ou administrations (frontières, géodésie, territoires, analyses thématique, imagerie verticale...)

La deuxième idée qui supporte l'approche processuelle de la cartographie est un prolongement de la réflexion sur la carte en tant que document sémiotique dynamiquement ouvert. L'ouverture ici signifie que l'idéal cartographique peut aussi être critiqué sur sa tendance à limiter l'appellation "carte" à des documents uniquement graphiques, matériels. En suivant la critique socioculturelle, on ne peut pas réduire la carte à sa matérialité : la carte est un « texte » qui peut être intégré à un discours plus large, fonctionner avec des éléments significatifs externes. Par exemple, un récit de voyage transmet des informations spatiales dont la partie graphique, sur une carte, ne constitue qu'un des aspects. Un cadastre comporte une partie graphique, mais cette dernière n'a pas de fonctionnement autonome, elle ne peut être utilisée seule. Les anthropologues rappellent aussi les traditions amérindiennes de transmission orale des connaissances spatiales.

11 Plus largement, l'auteur invite donc à prendre en compte les cartes dans leur contexte d'utilisation : leur interprétation est dépendante des circonstances dans lesquelles elles sont consommées, en lien avec d'autres "textes» au sens large, dans le cadre d'un réseau de communication, il s'agit donc d'un discours au sens linguistique. De plus, c'est au sein de discours précis qu'une carte peut acquérir une stabilité sémiotique, une signification récurrente, et lorsque l'on change de contexte ou de mode un document cartographique, il acquiert de nouvelles significations, par exemple lorsque des chants indigènes sont retranscrits dans le carnet d'un explorateur ou que des cartes marines sont utilisées pour la gestion de territoires terrestres. 
12 En conclusion de ce deuxième chapitre, l'auteur expose les conséquences de ce changement de point de vue pour les chercheurs, notamment d'un point de vue méthodologique. S'il existe une variété de pratiques de la cartographie, et que les cartes elles-mêmes n'ont de signification stable que dans leur contexte discursif particulier, alors le véritable sujet de la recherche en cartographie n'est pas la carte en tant qu'objet formel mais plutôt les démarches, les pratiques cartographiques qui les produisent, diffusent et consomment. Il faut alors s'intéresser à identifier ces usages cartographiques, les interpréter, observer leurs relations et leurs évolutions... En tant qu'historien, l'auteur insiste sur l'aspect dynamique de ces processus, pour lutter contre l'apparente stabilité des cartes promu par l'idéal cartographique, qui voudrait en faire des photographies de la réalité. Au contraire, les cartes, dans cette définition élargie qui s'établit progressivement, sont l'expression d'innombrables «actes cartographiques » (mappy acts, p. 48) : les concevoir, les diffuser, les utiliser, voire les ignorer...

13 Le troisième chapitre concentre la description du faisceau de préconceptions qui structurent l'idéal cartographique, via une liste claire et illustrée d'exemples. Elle a été constituée et remaniée au fil de l'expérience de l'historien (de nombreux exemples ou sous-composantes des préconceptions sont teintés par l'approche historique), mais il invite les lecteurs à étendre et amender ce travail. Les préconceptions sont souvent contradictoires, toutes sont fausses. Elles ont souvent déjà été individuellement repérées, mais de manière ponctuelle ou sans relever les relations ou dépendances avec d'autres préjugés, l'intérêt ici est que le regroupement soit systématique, synthétique et cohérent.

On n'en donnera ici qu'un résumé rapide, mais qui suffit à évoquer des exemples chez toute personne intéressée à la cartographie et son histoire.

- Ontologie : la carte est une réduction du monde, comparable à une base de données, selon un coefficient, l'échelle, qui indique directement le degré de la généralisation.

- Pictural : la carte est une représentation directe et mimétique du monde. C'est donc un document graphique ressemblant, qui utilise un langage graphique directement compréhensible et universel qui peut même rendre visible des phénomènes abstraits.

- Individualité : la conception et l'utilisation des cartes sont des actes individuels. Construire des représentations spatiales mentales est un processus fondamentalement humain et rationnel. En conséquence, l'aspect d'une carte peut renseigner sur la capacité mentale de son concepteur/réalisateur (!), et on peut juger les peuples, les genres ou les groupes sociaux par la qualité de leurs productions cartographiques (re-!).

- Matérialité : les cartes sont des objets, produits à un instant précis et autonomes. Il s'en suit que la cartographie peut être décomposée en deux moments distincts: la fabrication et l'utilisation des cartes.

- Observation: les cartes sont toutes issues de l'observation et de la mesure du réel, de la nature, depuis un point de vue nadiral (du haut vers le bas) et objectif (sans prise en compte de l'expérience de l'observateur). On pourra ici évoquer l'utilisation figurée du mot "cartographie ", exprimant une observation méticuleuse et si possible exhaustive d'un phénomène, qui semble se développer en français (cf. les travaux sur les métaphores spatiales).

- Efficacité / opérationnalité : les cartes sont des outils, des instruments, spécialement pour guider les déplacements. Toutes les cartes peuvent être utilisées pour se repérer et se 
déplacer, et elles l'ont toujours été. Les cartes sont le meilleur outil pour décrire les relations spatiales et guider les déplacements.

- Discipline (au sens de la formation à de bonnes pratiques, de règles de conduite) : les cartes sont vérifiées et améliorées par la comparaison avec la réalité. Les cartes sont régulièrement mises à jour et on peut mesurer l'importance d'une carte par le nombre de ses mises à jour. Lorsque de nouvelles données, de nouveaux relevés sont réalisés, les cartes sont mises à jour. On peut donc dater une carte de la plus récente entité qu'elle représente. La cartographie est une opération essentiellement positiviste, progressiste, la quantité et la qualité de l'information ne peuvent qu'augmenter avec le temps.

- Publicité : Les cartes sont faites pour être diffusées largement. Les cartes imprimées sont plus efficaces et plus importantes que les cartes manuelles, souvent seulement des témoins des opérations préliminaires (relevés).

- Moralité : la production de cartes et un acte essentiellement moral, marque de progrès. Les cartographes se doivent de produire les meilleures cartes possibles, en suivant une bonne discipline (cf. plus haut). Les mauvaises cartes sont imprécises et immorales. La société veille à réguler et discipliner la cartographie, qui est un indice de la qualité et de la compétence d'une nation entière.

- Unicité et universalité : la cartographie est une activité unique pratiquée par tous les cartographes, pour un seul monde/archive, avec une seule technologie d'observation, un seul objectif. La cartographie est une science, elle possède des normes reconnues et universelles, on peut parler de « raison cartographique ».

Chacune de ces préconceptions est ensuite expliquée, détaillée et illustrée dans la suite du troisième chapitre, sur près de cinquante pages. M. H. Edney utilise de nombreuses citations d'ouvrages ou d'articles scientifiques qui démontrent à quel point ces préconceptions ont pu être répandues et plus ou moins prescriptives. La très conséquente bibliographie présentée dans ce chapitre constitue une revue de littérature appréciable. Si les préconceptions ont été détectées et décrites par un travail d'historien et d'épistémologue de la cartographie, on en trouve néanmoins toujours des traces et des effets dans les activités actuelles. L'évolution des définitions scientifiques de la cartographie est à ce sujet assez parlante. L'auteur développe des thèmes transversaux au croisement de plusieurs préconceptions, comme les idées racistes ou sexistes qui ont pu se développer ou être implicites de productions cartographiques.

Les (volumineux) chapitres suivants vont être l'occasion pour l'auteur de détailler son approche en mettant en pratique la grille de lecture des préconceptions dans une optique historique, pour mettre au jour et démonter des combinaisons de préconceptions particulièrement pernicieuses, d'un point de vue épistémologique mais aussi plus largement pour la culture et l'histoire des sociétés.

Le quatrième chapitre va s'intéresser à l'histoire de l'émergence progressive de l'idéal cartographique en observant la construction par la société occidentale de la cartographie comme une science apparemment cohérente, morale et universelle basée sur l'observation et la mesure. Le croisement de l'évolution technique, des besoins de connaissance précise de nouveaux territoires (colonies) pour un meilleur contrôle, mais aussi des routes maritimes, de l'intégration des documents cartographiques dans des archives spécialisées, de la diffusion publique par l'impression, sont bien connus des historiens de la cartographie, mais prennent ici un nouveau jour: la liste des préconceptions devient une grille d'analyse qui guide l'interprétation et permet de croiser les hypothèses. La prégnance de l'idéal dans la culture moderne s'explique en 
bonne part, selon l'auteur, par l'ancienneté et la progressivité de son développement. Les scientifiques, en particulier, ont hérité d'un idéal existant et structuré par des convictions bien implantées, renforcées et nuancées par de nouveaux facteurs et usages (comme le développement de l'automobile et des cartes routières, puis du GPS, de l'informatique et des observations par satellite). Par ailleurs, comme on l'a vu au chapitre précédent au travers des contradictions de certaines préconceptions, internes comme entre elles, l'idéal n'est pas un ensemble logique construit : il est un assemblage multi-facettes de différentes croyances et convictions qui possède « une remarquable résilience interne » (p. 164).

Le chapitre s'achève par des observations très intéressantes concernant des tendances plus récentes, par exemple sur le développement d'une cartographie plus populaire ou moins sérieuse (voire humoristique et caricaturale), plus métaphorique que précise, notamment observée par les chercheurs qui ont suivi B. Harley et la critique « socioculturelle » sur les aspects rhétoriques de la cartographie. Cette critique, si elle a permis de considérables progrès pour la recherche cartographique récente, reste sous l'influence des préconceptions évoquées, notamment l'idée que la cartographie constitue une entreprise unique, monolithique et progressiste.

Le chapitre cinq s'intéresse justement en détail à un élément central de l'idéal cartographique moderne: l'échelle. A priori simple, mathématiquement comme conceptuellement, le concept d'échelle a progressivement vu sa signification évoluer, particulièrement dans ses connotations, ses implicites. Tout d'abord, le simple de fait de proposer une échelle fixe pour tout le document cartographique implique que la projection soit maitrisée et le permette. Ainsi, les représentations en vue oblique ou en anamorphose sont automatiquement évacuées. Par l'action des archivistes et documentalistes à la recherche de moyens de classification, l'échelle en est venue à exprimer assez directement le degré de généralisation de la carte, la quantité et la proportionnalité de son contenu (par rapport au réel). Cette réduction est, forcément, impossible et toujours fausse. Ce problème s'est vu accru par la simplification de la notion d'échelle sous la forme d'un simple ratio $1 / \mathrm{X}$.

Le dernier chapitre commence par une synthèse de l'évolution de l'idéal cartographique en quatre périodes historiques et se poursuit par un point sur son étendue actuelle et sur les moyens de lutter contre ses préconceptions, qui est tout particulièrement intéressant. Depuis les années 1970, avec le développement de la critique «socioculturelle » et ce que l'on a pu appeler la «démocratisation » de la production des cartes (plus grande accessibilité des données et des outils, plus grande diffusion dans la presse et sur Internet), l'idéal cartographique est mieux identifié, remis en question. L'auteur cite l'article provocateur de D. Wood en 2003: "Cartography Is Dead (Thank God!)» (au sens où le mapping se développe, la production cartographique échappe à « l'étreinte mortifère des scientifiques » et n'est plus le monopole des agences publiques).

21 M. H. Edney termine son ouvrage en rappelant son objectif (p. 234): démontrer les nombreux défauts et l'artificialité de l'idéal cartographique. Le livre n'y arrivera pas à bout mais l'auteur espère avoir contribué à l'affaiblir sévèrement. Il propose des pistes pour poursuivre le travail, notamment s'intéresser plus spécifiquement aux utilisateurs des cartes, dans leur diversité, ainsi qu'aux diverses "modes " cartographiques qui peuvent produire des documents sémiotiques eux aussi très variés. Il rappelle (chap. 2) que la carte est un discours qui suppose des producteurs et des consommateurs 
possédant un large panel de stratégies et de moyens de communication. L'objet de la recherche cartographique doit être orienté vers l'analyse de ces discours spatiaux, de leur variété, pour aider à leur interprétation, à la mesure de leur rôle dans les sociétés humaines, dans le passé, le présent et le futur.

Après une telle lecture, si remuante, on peut ressentir un certain étourdissement, notamment grâce à la force et à la forme des arguments présentés, qui invitent à l'identification des préconceptions et l'évocation d'exemples propres chez tout familier des cartes.

On peut néanmoins relever quelques éléments qui nous laissent un peu sur notre faim. Tout d'abord, le texte est abondamment muni d'exemples (et parfois d'images), mais, à cause de l'expérience de l'auteur et de sa volonté d'évoquer l'émergence de l'idéal, ces exemples sont principalement tirés de périodes historiques relativement anciennes (XVII ${ }^{\mathrm{e}}$-XVIII ${ }^{\mathrm{e}}$ siècles) et concernent pour beaucoup la cartographie nord-américaine. Par conséquent, on ressent un manque tout particulier de réflexion sur les dernières décennies, qui ont vu une majorité des "modes" cartographiques connaître une révolution, et qui ne sont que relativement peu évoquées en fin d'ouvrage. Ainsi, on pourrait aisément compléter la liste de ces «modes » présente au chapitre 2 (p. 33) et celle des préconceptions, ou du moins fournir des avatars récents à certaines d'entre elles, tirées de l'observation de la cartographie participative, du géo-web, de l'expression artistique, de nouveaux usages... Ce qui permettrait de poursuivre le travail et d'en améliorer la pertinence pour les chercheurs intéressés aux techniques et pratiques actuelles. Si la portée épistémologique des arguments de M. H. Edney est clairement sensible, les applications pourtant potentiellement majeures sur les pratiques et les usages actuels sont en retrait.

Enfin, on l'a évoqué en début de compte-rendu, le repérage et la critique de l'idéal cartographique sont gênés dans les langues qui ne possèdent qu'un seul mot pour décrire la production de cartes, le (relatif) néologisme de "cartographie ». Il serait intéressant d'en faire une étymologie critique en français et de repérer les usages « normatifs » du mot, avant d'envisager de mettre à jour les dictionnaires scientifiques.

\section{AUTEURS}

\section{LAURENT JÉGOU}

Université de Toulouse-Jean Jaurès, UMR LISST-Cieu 